\title{
Feasibility and Efficacy of Bright Light Therapy in Depressed Adolescent Inpatients
}

\author{
First Open-Label, Single-Arm, Prospective Clinical Trial \\ Inken Kirschbaum-Lesch¹, Stephanie Gest ${ }^{1}$, Tanja Legenbauer ${ }^{1, a}$, and Martin Holtmann,a \\ Ruhr-University Bochum, LWL-University Hospital for Child and Adolescent Psychiatry and Psychotherapy, Hamm, Germany \\ a Contributed equally to the manuscript and study.
}

\begin{abstract}
Objective: Bright light therapy (BLT) has recently come into increasing focus in the treatment of adolescent depression, whereby light glasses today appear to be more feasible than light therapy boxes. This study investigated the feasibility and efficacy of 4 weeks of BLT with light glasses. It also analyzed whether a treatment duration of 4 weeks of BLT yields larger effects than the 2 weeks of BLT investigated in previous studies. Methods: This first open-label, single-arm, prospective clinical trial pursued a naturalistic approach: 39 inpatients aged 12-18 years with moderate or severe depression received 4 weeks of morning BLT with light glasses in addition to usual treatment. Depressive symptoms, sleep problems, circadian phase, and the clinical global impression were assessed at several timepoints. In a second analysis, the data of the present study were compared to those from a previous pilot trial. Results: Depressive symptoms, sleep problems, and the global clinical impression improved significantly after BLT with light glasses, whereas the circadian phase did not change over time. Light glasses showed similarly positive effects on sleep parameters and depressive symptoms as light boxes. Contrary to expectation, prolonging BLT to 4 weeks did not yield larger effects on depressive symptoms and sleep complaints compared to 2 weeks of intervention. Conclusions: Light glasses seem to be a feasible and highly acceptable method for the treatment of adolescent depression. Further randomized controlled trials are needed to obtain sufficient evidence regarding the efficacy of BLT as an add-on intervention to psychological and pharmacological approaches for adolescent depression.
\end{abstract}

Keywords: bright light therapy, adolescents, depression, sleep, light glasses

\section{Introduction}

Bright light therapy (BLT) is well established as an additional method to support psychotherapeutic and psychopharmacological approaches in the treatment of depressed adults (Al-Karawi \& Jubair, 2016). Recent meta-analytic evidence showed a significant reduction of depressive symptoms for nonseasonal depression after BLT administered for 1-8 weeks (Al-Karawi \& Jubair, 2016). BLT synchronizes the sleep-wake rhythm, which is often disturbed in patients with sleep and mood disorders (Wirz-Justice et al., 2013). Morning BLT suppresses the secretion of melatonin and leads to an increased wakefulness during the day as well as to an advanced sleep phase, whereas evening BLT leads to a delayed sleep phase. Furthermore, it is assumed that BLT increases serotonergic and noradrenergic levels, resulting in improved mood (e.g., Stephenson, Schroder, Bertschy, \& Bourgin, 2012).
Controlled trials showing the effectiveness of BLT previously used UV-filtered broad-spectrum white light of about 10,000 lux. This light intensity is comparable to skylight 40 min after sunrise (Wirz-Justice et al., 2013). Duration of exposure depends on the light intensity. With 10,000 lux, exposure times of $30 \mathrm{~min}$ are recommended (Wirz-Justice et al., 2013), whereas lower light intensities need longer exposure times. To date, the optimal treatment duration of BLT is unknown. In their meta-analysis, Al-Karawi and Jubair (2016) concluded that the best effectiveness was reached when BLT was applied for 2-5 weeks.

Previous studies used BLT therapy boxes with different light intensities. Patients sat in front of a BLT lamp and were instructed to look into the light from time to time (e.g., Gest et al., 2015). This method has some limitations, as patients are less flexible during the time of the intervention: They have to sit in front of the lamp for 30-45 min, mostly before school, which may be difficult to integrate 
into their daily routine and can also be uncomfortable and tiring. Light glasses, which encompass light that falls onto the lower half of the eyes without the need to look directly into the light, may reduce some of these limitations. Patients wearing these glasses are able to go about their daily activities, such as reading, listening to music, or having breakfast. One study investigated the effects of light therapy glasses compared to light boxes and dim light placebo on visual comfort, mood, alertness, concentration, and sleep quality in 24 healthy young adults aged 20-35 years (Viola et al., 2014). The results suggest that light therapy glasses seem to be as efficient as light boxes.

Studies investigating the effects of BLT on depressive symptoms in children and adolescents are scarce. Pilot studies provide evidence for the effectiveness of BLT, especially for the reduction of sleep complaints. The first randomized controlled trials revealed the superior effects of BLT in reducing depressive symptoms in an active treatment group (1-2 weeks of BLT) compared to placebo in children and adolescents (aged 7-17 years; Niederhofer \& von Klitzing, 2011; Swedo et al., 1997). Another randomized controlled trial in an inpatient setting compared morning BLT (10,000 lux) to dim light placebo (100 lux) in 58 children and adolescents (aged 12-18 years; Bogen et al., 2016). Morning BLT was applied for 45 minutes and over 2 weeks. The authors found no direct effects of BLT on depressive symptoms, as a significant reduction of depressive symptoms emerged in both groups, though stable improvements in sleep parameters emerged only in the active BLT group. In addition, morning BLT led to a circadian phase shift toward morningness as measured with a chronotype questionnaire, whereas dim light placebo did not show such an effect. The reduction of depressive symptoms was predicted by the improvements in sleep quality and the circadian phase shift toward morningness.

Support for these findings was provided by Gest et al. (2015), who compared a combination of BLT and a night of sleep deprivation to morning BLT only in depressed inpatient adolescents (aged 13-18 years) and found a positive influence of BLT on sleep parameters but only indirect effects on the reduction of depressive symptoms. Contrary to expectation, a night of sleep deprivation had no additional effect. A secondary analysis of the data using actigraphy and sleep diary data showed a sleep phase advance in the second week of intervention in the BLT only group (Kirschbaum et al., 2017).

In summary, 2 weeks of BLT with light boxes repeatedly proved to significantly improve sleep problems in adolescents, while data on the antidepressant effects are not yet sufficient. Some lines of evidence suggest that the effects of BLT need some time to develop, and that efficacy of BLT increases with longer duration of intervention (e.g., AlKarawi \& Jubair, 2016; Martiny et al., 2015). In addition, the equivalence of light boxes with light glasses has not yet been studied in youths. The present study proposed to study the effects of BLT with light glasses, while prolonging the duration of exposure to 4 weeks.

The aims of the present study were as follows:

1. to investigate the feasibility of light glasses and the effects of 4 weeks of BLT with light glasses on depressive symptoms, sleep, chronotype and clinical global impression;

2. to compare the effects of light glasses from the present study (LUM - bright light therapy with light glasses which are called „Luminette ${ }^{\circledR(")}$ after 2 weeks of intervention on depressive symptoms and sleep parameters to those of light boxes (LT-box) using data from a light therapy group of a previous pilot trial conducted by our work group (Gest et al., 2015);

3. to compare effects of 4 weeks of BLT (LUM) to those of 2 weeks of BLT (LT-box) 4 weeks after the start of BLT.

It was our assumption that light glasses have effects comparable to those of light boxes after 2 weeks of treatment, but that light glasses are more feasible and flexible in the inpatient setting than light boxes. In addition, we hypothesized that prolonging treatment duration to 4 weeks would lead to larger effects than a 2 -week intervention.

\section{Methods}

\section{Study Design and Ethical Standards}

The present open-label, single-arm, prospective clinical trial pursued a naturalistic approach. Depressed inpatients received 4 weeks of BLT with light glasses additional to treatment as usual (TAU). Depressive symptoms, sleep problems, chronotype, and the global clinical impression were assessed via questionnaires before the intervention (T1), after 2 weeks of intervention (T2), directly after the intervention (T3), and 2 weeks after the end of intervention (T4). The data of the present study (LUM) were compared to those of a randomized control trial (LT-box) in which BLT was administered for 2 weeks with a light therapy box (Gest et al., 2015).

The previous study with light therapy boxes was approved by the Ethics Committee of the Medical Faculty at the Ruhr-University Bochum (registration number 4418-12). The light therapy glasses were used in the clinical routine, and patients and their caregivers signed an informed consent document for using the collected routine data. The collection of routine data was also approved by the medical faculty's Ethics Committee. The study was conducted in accordance with the ethical principles of the Declaration of Helsinki in its current version. 


\section{Sample}

A total of 51 depressed inpatients at the LWL-University Hospital Hamm (Germany) were recruited from January 2016 to February 2017 and were assessed for eligibility. Thereof, 12 patients had to be excluded: 11 did not meet the inclusion criteria (e.g., a BDI-II score <20), and one patient refused to participate. Therefore, 39 inpatients participated in this study (for an overview, see Figure 1). Inclusion criteria were (1) moderate to severe depressive symptoms, as assessed on the BDI-II, (2) age between 12 and 18 years, and (3) a planned inpatient stay of at least 4 weeks. Patients were excluded if they (1) had been diagnosed with schizophrenia, showed psychotic symptoms or suicidal tendencies; (2) were being treated with antipsychotics or beta blockers; (3) were pregnant; (4) were hypersensitive to light or had eye diseases. Because this was a naturalistic setting, antidepressant medication was allowed and controlled for in the analyses performed. All patients participated voluntarily, and permission was granted both by them and their caregivers.

The comparison group consisted of 37 depressed inpatients of the LWL-University Hospital Hamm (Germany) aged 13-18 years who received 2 weeks of BLT via therapy boxes. These patients were part of a randomized control trial in which a combination of wake therapy and BLT was compared to morning BLT only (Gest et al., 2015). The previous study had the same inclusion and exclusion criteria (as described above). For a more detailed description see Gest et al. (2015).

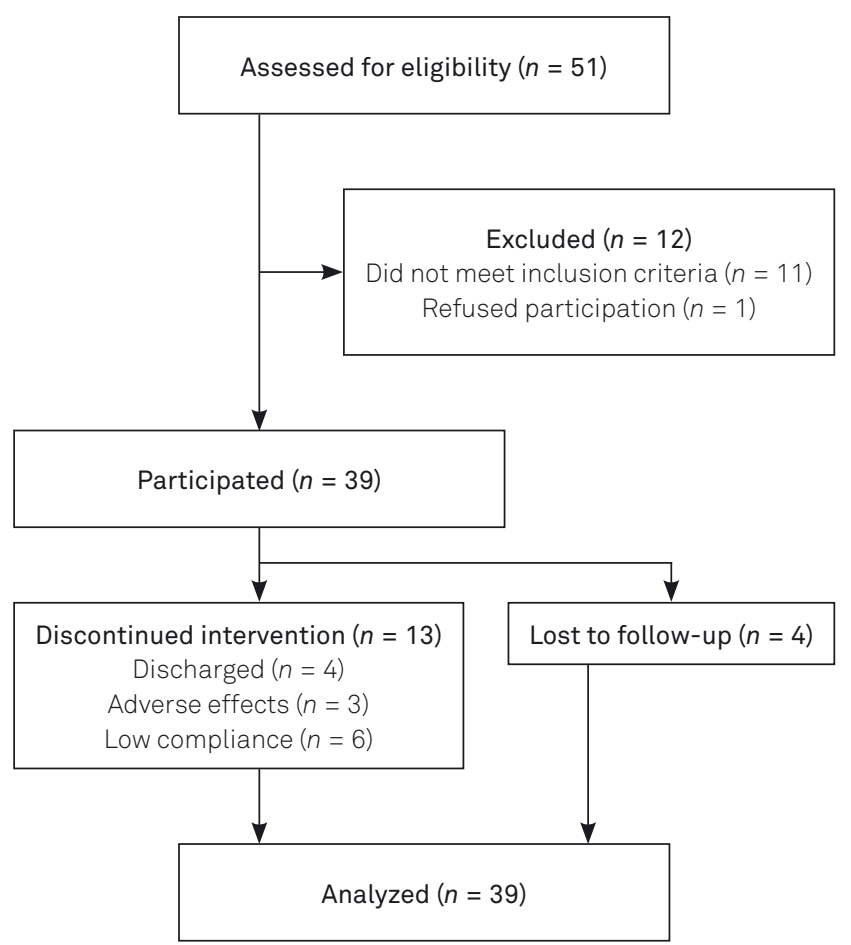

Figure 1. Patient flow.

\section{Variables and Measures}

\section{Beck Depression Inventory-II (BDI-II)}

The BDI-II (Beck et al., 1996) is a 21-item self-report instrument for the assessment of depressed mood during the previous 2 weeks. The German version of the BDI-II showed good validity and internal consistency in samples of both adolescents and adults (Kühner et al., 2007; Besier et al., 2008). The presence of a moderate depressive episode is indicated by a score of $>19$, and the presence of a severe depressive episode is indicated by a score of $>28$. The BDIII showed good psychometric properties in the current sample (Cronbach's $\alpha=0.81$ ).

\section{Sleep}

Sleep experiences and sleep habits were assessed by the well-established and validated German sleep questionnaire "Schlaffragebogen B revised" (SF-BR; Görtelmeyer, 2011). The SF-BR consists of 31 items ( 6 factor scales and 5 sleep indexes) and assesses sleep characteristics during the previous 2 weeks. The SF-BR revealed moderate to excellent internal consistency $(\alpha=.68-.92)$ within clinical samples and a test-retest reliability from $r=.53$ to $r=.91$. Based on pilot studies by our work group (Bogen et al., 2016; Gest et al., 2015), the present study focused on two scales: "Sleep quality" (SF-SQ) and "Awaking after restorative sleep" (SF-FOR). SF-SQ consists of the indexes "waking at night," "difficulty falling asleep," "premature awakening," "general sleep characteristics," and the item "sleep in an unfamiliar environment." The SF-FOR includes items concerning feelings after waking up (e.g., "feeling refreshed after awakening"). Healthy adolescents and adults (aged 16-32 years) showed a mean score of 3.94 (0.62) for SF-SQ and $3.12(0.75)$ for SF-FOR.

\section{Morningness-Eveningness Questionnaire (MEQ)}

The German version of the Morningness-Eveningness Questionnaire (D-MEQ; Horne \& Östberg, 1976) used 15 items to assess circadian preferences and circadian phase shift. With the sum score, respondents can be classified into one of five groups: "definitely morning type," "moderate morning type," "definitely evening type," "moderate evening type," and "neutral type." The questionnaire is suitable for both adolescent and adult samples. The D-MEQ was also used to determine the ideal time slot for BLT. The MEQ correlates with the dim light melatonin onset (DLMO) $(r=$ -.73), which represents the onset of melatonin secretion and is seen as the gold standard for characterizing the individual circadian rhythm (Wirz-Justice et al., 2013).

\section{Clinical Global Impression (CGI) Scale}

The CGI was used to assess the global severity of the symptoms (CGI-S) at baseline and the global change in illness 
compared to baseline (CGI-I). The CGI-S is rated on a 7-point scale from $1=$ normal, not at all ill to 7 = among the most extremely ill patients. The CGI-I is also rated on a 7-point scale, from 1 = very much improved since the intervention treatment to $7=$ very much worse since the intervention treatment. The CGI was filled out by the attending therapist.

\section{Adverse Events}

Adverse events (e.g., headache, dizziness) are generally rare, mild, and short-lasting among those receiving BLT (Wirz-Justice et al., 2013). However, patients were informed about and instructed to observe possible adverse events. They were assessed on a weekly basis using open questions.

\section{Light Therapy Devices}

Luminette $^{\circledast}$ (Lucimed, Belgium) light therapy glasses radiating blue light $(468 \mathrm{~nm})$ with a power of 10,000 lux were used. These glasses were compared to the light therapy box PhysioLight LD1100 (DAVITA) used in the study by Gest et al. (2015), which radiates white light of 10,000 lux at a distance of $65 \mathrm{~cm}$.

\section{Procedures}

The hospital admission list was checked several times a week for patients diagnosed with moderate or severe depression. These patients were screened for eligibility and were informed about the study. After the patients and their caregivers had provided written informed consent, the pretest (T1) was conducted. The patients completed questionnaires, were familiarized with the light glasses and were informed about possible adverse events. In addition, the ideal time slot for BLT was determined using the MEQ. The intervention began on the following day. The inpatients received 4 weeks of morning BLT for 30 minutes, with weekends being excluded. After 2 weeks of intervention, an interim assessment (T2) was conducted, and after 4 weeks of intervention, the posttest (T3) was carried out. A follow-up assessment (T4) was performed 2 weeks after the end of the intervention.

\section{Statistical Methods}

All analyses were based on an intention-to-treat (ITT) population comprising all included patients. Missing values were replaced by multiple imputation. First, changes in depressive symptoms, sleep, chronotype, and clinical global impression were investigated using repeated measures ANOVAs with time (T1, T2, T3, T4) as within-subject factor. Further posthoc analyses with paired $t$-tests were performed to identify significant differences between the four timepoints. To determine whether light therapy glasses lead to the same effects as light therapy boxes, and to investigate whether prolonging treatment duration to 4 weeks of BLT leads to larger effects, the data of the present study (LUM) were compared to those from the BLT group of the study by Gest et al. (2015; LT-box). For the analyses, difference scores from $\mathrm{T} 1$ to $\mathrm{T} 2$ and from $\mathrm{T} 2$ to $\mathrm{T} 3$ were computed for the BDI-II, SF-SQ, and SF-FOR. Then, univariate ANOVAs for the variables depressive symptoms and sleep were conducted. The two-sided level of significance was set at $\alpha=$ .05. All data were analyzed using SPSS 24.0 for Windows.

\section{Results}

\section{Sample Characteristics}

Of the 39 patients included, 32 (82.1\%) were female and the mean age was $15.74(1.14(\mathrm{SD}=1.14))$ years. Thirty-two $(82.1 \%)$ participants indicated severe depressive symptoms, assessed by means of the BDI-II. The global severity of the symptoms (CGI-S) was predominantly categorized by the attending therapist as "patient is clearly sick" (76.9\%). Seven (17.9\%) were categorized as "patient is only moderately sick," and two (5.1\%) as "patient is seriously sick." With regard to chronotype, 10 (25.6\%) patients were categorized as "definitely evening," 14 (35.9\%) as "moderate evening," 14 (35.9\%) as "neutral," and one $(2.6 \%)$ as "moderate morning," as assessed on the DMEQ. One-third of the patients reported headache and dizziness, which often occurred at the beginning of the intervention, but was short-lasting. Eye irritability, nausea, and seeing spots in front of the eyes were rarely reported.

Sample characteristics of both groups are displayed in Table 1. No statistical differences were found with respect to the reported parameters. The two samples also did not

Table 1. Descriptive statistics and comparisons of the baseline parameters by groups

\begin{tabular}{lccccc}
\hline & $\begin{array}{c}\text { LUM } \\
(n=39)\end{array}$ & $\begin{array}{c}\text { LT-box } \\
(n=35)\end{array}$ & F & $\chi^{2}$ & $\mathrm{p}$ \\
\cline { 2 - 5 } & $M(S D)$ & $M(S D)$ & & & \\
\hline Age (years) & $15.74(1.13)$ & $15.34(1.07)$ & 0.87 & .13 \\
CGl-S & $4.87(0.48)$ & $4.7(0.6)$ & 2.33 & .23 \\
\hline & $n(\%)$ & $n(\%)$ & & & \\
\hline Sex ratio (female) & $32(82.1 \%)$ & $33(94.3 \%)$ & & 2.58 & .16 \\
Antidepressant & $14(35,9 \%)$ & $13(37.1 \%)$ & 0.001 & .98 \\
medication & & & & \\
\hline
\end{tabular}

Note. $\mathrm{CGI}-\mathrm{S}=$ Severe score of the Clinical Global Impression Scale. 
differ with respect to BDI-II sum scores (LUM: $M=37.46$, LT-box: $M=36.91, p=.97)$, SF-SQ (LUM: $M=2.43$, LT-box: $M=2.61, p=.42$ ), and SF-FOR (LUM: $M=2.08$, LT-box: $M=2.05, p=.62)$. For a more detailed description of the clinical and demographical sample characteristics of the comparison group, see Gest et al. (2015).

\section{Changes in Depressive Symptoms, Sleep, Chronotype, and Clinical Global Impression (LUM Group)}

Baseline scores (T1) and changes in depressive symptoms, sleep, chronotype and clinical global impression at $\mathrm{T} 2$, T3, and T4 are shown in Table 2. Depressive symptoms changed significantly over time, $F(3,114)=7.68, p=$ $.001, \eta^{2}=.168$, with posthoc analyses revealing significant improvements from T1 to T2 $(p=.015)$. For sleep, a main effect of time was found, $F(3,114)=4.45, p=.017$, $\eta^{2}=.105$ : Sleep improved significantly from T1 to T3 ( $p=$ .018). No significant main effect of time was identified for chronotype, although there was a difference at the trend level between T1 and T2 $(p=.057)$. The clinical global impression improved significantly over time, $F(2,48)=$ $7.42, p=.002, \eta^{2}=.24$, with posthoc analyses revealing significant improvements from T2 to T4 $(p=.004)$ and from $\mathrm{T} 3$ to $\mathrm{T} 4(p=.007)$.

\section{Comparison Between Light Glasses and Light Boxes after 2 Weeks of Intervention (LUM vs. LT-Box)}

Changes from $\mathrm{T} 1$ to $\mathrm{T} 2$ in depression, sleep quality, and awaking after restorative sleep did not differ between the two groups (BDI-II: $F(1,73)=1.10, p=.299$; SF-SQ: $F(1$, $72)=.28, p=.60 ;$ SF-FOR: $F(1,73)=.44, p=.51$; calculated with the difference scores from $\mathrm{T} 1$ to $\mathrm{T} 2$ ).

\section{Comparison Between 2 and 4 Weeks of BLT (LUM vs. LT-Box)}

The effects of 4 weeks of BLT did not differ from those of 2 weeks of intervention with respect to the BDI-II sum score, $F(1,54)=.08, p=.78$, SF-SQ, $F(1,73)=.52, p=.47$, and SF-FOR, $F(1.73)=.48, p=.49$ (calculated with the difference scores from $\mathrm{T} 2$ to $\mathrm{T} 3$ ). Changes in depressive symptoms, SF-SQ, and SF-FOR from T1 to T3 are displayed in Figure 2.

\section{Discussion}

The present open-label, single-arm, prospective clinical trial investigated the feasibility and efficacy of 4 weeks of BLT with light glasses in depressed adolescent inpatients as well as comparing light glasses to light boxes. As expected, light glasses showed similar effects on depressive symptoms and sleep parameters as those of light boxes. Regardless of the application method (glasses or boxes), 2 weeks of BLT led to significant improvements in sleep quality and depressive symptoms. However, the hypothesis that a longer duration of BLT leads to greater effects was not confirmed: Reduction of depressive symptoms and sleep problems 4 weeks after the start of BLT did not differ between inpatients who had received 4 weeks of BLT (LUM) and those who had received 2 weeks of BLT (LT-box).

BLT has recently come into increasing focus as an addon intervention in the treatment of adolescent depression. Pilot studies provided first evidence that BLT positively influences sleep complaints and depressive symptoms in nonseasonal depression (e.g., Bogen et al., 2016; Gest et al., 2015). These studies used light therapy boxes, which are limited by their inflexibility and inconvenience. The present study in turn used light glasses, which were highly accepted both by the adolescents and their caregivers. The

Table 2. Descriptive means $M$ and standard deviations SD at various assessments

\begin{tabular}{|c|c|c|c|c|c|}
\hline & BDI-II & SF-SQ & SF (FOR) & D-MEQ & CGI-I \\
\hline & $M(S D)$ & $M(S D)$ & $M(S D)$ & $M(S D)$ & $M(S D)$ \\
\hline T1 & $37.46(8.46)$ & $2.43(0.85)$ & $2.08(0.63)$ & 34.33 (9.87) & \\
\hline T2 & $31.13(14.74)$ & $2.65(0.83)$ & $2.21(0.77)$ & 35.95 (10.69) & $3.22(0.66)$ \\
\hline T3 & $30.30(19.11)$ & $3.48(1.79)$ & $2.32(0.82)$ & $36.06(13.01)$ & $3.23(0.73)$ \\
\hline T4 & $23.57(18.36)$ & 3.38 (2.52) & $2.84(1.88)$ & $40.49(29.76)$ & $2.85(0.86)$ \\
\hline
\end{tabular}

Notes. BDI-II = Beck Depression Inventory-II, SF-SQ = scale sleep quality in the questionnaire SF-BR, SF-FOR = scale awaking after restorative sleep in the questionnaire SF-BR, D-MEQ = German version of the Morningness-Eveningness Questionnaire, CGI-I = Clinical Global Impression-Improvement, T1 = pre-test, T2 = interim assessment, $\mathrm{T} 3=$ post-test, $\mathrm{T} 4$ = follow-up assessment two weeks after the end of intervention. 

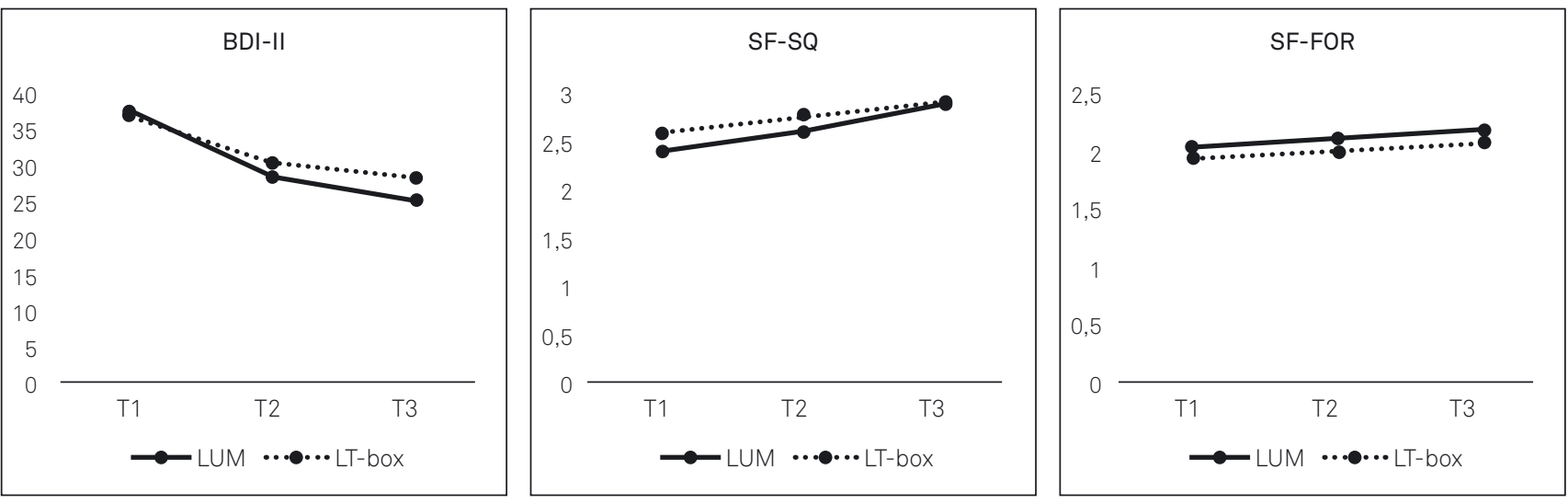

Figure 2. Changes in depressive symptoms and sleep variables from T1 to T3. BDI-II = Beck Depression Inventory-II, SF-SQ = Scale Sleep Quality in the questionnaire SF-BR, SF-FOR = Scale Awaking after Restorative Sleep in the questionnaire SF-BR, LUM = bright light therapy with light glasses, LT-box = bright light therapy with light box.

light glasses were easier to integrate into daily routines and the routines of the hospital ward. Besides exhibiting improved depressive symptoms and sleep, the inpatients showed an improved clinical global impression and a marginally significant shift in chronotype toward morningness. The latter was also found in a pilot study using light boxes (Bogen et al., 2016). Because the present study applied BLT for 4 weeks (in addition to TAU for depressed adolescent inpatients), it might be argued that it is difficult to differentiate which form of therapy caused which effects. However, in the pilot study by Bogen et al. (2016), only the BLT group showed significant improvements in sleep parameters, while the placebo group did not.

Contrary to first evidence in adults, which suggests that longer treatment durations of BLT yield larger effects (AlKarawi \& Jubair, 2016; Martiny et al., 2015), the present study found no difference between 2 and 4 weeks of BLT. Al-Karawi and Jubair (2016) pointed out that studies that systematically investigate the duration of BLT in adults as well are still lacking, and further research is clearly needed. Because it may take some time for BLT to reach a certain level of effectiveness (Kennedy et al., 2001), follow-up assessments are necessary to measure the longterm effects of BLT. Additionally, the present sample size may not have had sufficient power to detect small effects.

To the best of our knowledge, this is the first study to investigate the efficacy of 4 weeks of BLT and of light glasses in depressed adolescent inpatients. The strengths of the study lie in the naturalistic approach and the high acceptance of light therapy with light glasses for the treatment of adolescent depression. However, some limitations should be taken into account. First of all, because this was a pilot study investigating the feasibility of light glasses, it was not possible to include a control group. A second limitation is the high dropout rate (one-third of participants), which can be explained by the naturalistic setting (several patients were discharged before the BLT was completed) and the high personal responsibility of the inpatients to remember to wear the glasses every morning. Because depressed inpatients, especially those with a severe episode, can have symptoms such as anhedonia, they might need more support and motivation, which should be considered in future studies.

Overall, this pilot study showed that light glasses are as effective as a 10,000 lux light box after 2 weeks of treatment. Prolonging BLT to 4 weeks did not yield larger effects on depressive symptoms and sleep complaints. Further randomized controlled trials with large sample sizes are necessary to obtain sufficient evidence regarding the efficacy of BLT as an add-on intervention to psychological and pharmacological approaches in the treatment of depressed adolescents and regarding the required treatment duration.

\section{References}

Al-Karawi, D., \& Jubair, L. (2016). Bright light therapy for nonseasonal depression: Meta analysis of clinical trials. Journal of Affective Disorders, 198, 64-71.

Beck, A.T., Steer, R.A., \& Brown, G. K. (1996). Beck Depression Inventory Manual. San Antonio: TX: The Psychological Corporation.

Besier, T., Goldbeck, L., \& Keller, F. (2008). Psychometric properties of the Beck depression inventory-II (BDI-II) among adolescent psychiatric patients. Psychotherapie Psychosomatik Medizinische Psychologie, 58(2), 63-68.

Bogen, S., Legenbauer, T., Gest, S., \& Holtmann, M. (2016). Lighting the mood of depressed youth: Feasibility and efficacy of a 2 week, placebo-controlled bright light treatment for juvenile inpatients. Journal of Affective Disorders, 190, 450-456.

Gest, S., Holtmann, M., Bogen, S., Schulz, C., Pniewski, B., \& Legenbauer, T. (2015). Chronotherapeutic treatments for depres- 
sion in youth. European Child and Adolescent Psychiatry, 25, 151-161.

Görtelmeyer, R. (2011). Manual zum SF-A/R und SF-B/R - Schlaffragebogen A und B - Revidierte Fassung. Gießen: Hogrefe.

Horne, J.A., \& Östberg, O. (1976). A self-assessment questionnaire to determine morningness-eveningness in human circadian rhythms. International Journal of Chronobiology, 4, 97-110.

Kennedy, S. H., Lam, R. W., Cohen, N. L., Ravindran, A.V., \& CANMAT Depression Work Group. (2001). Clinical guidelines for the treatment of depressive disorders. IV. Medications and other biological treatments. The Canadian Journal of Psychiatry, 46, 38-58.

Kirschbaum, I., Straub, J., Gest S., Holtmann, M., \& Legenbauer T (2017). Short-term effects of wake- and bright light therapy on sleep in depressed youth. International Journal of Chronobiology, 35 (1), 10.1080/07420528.2017.1388251.

Kühner, C., Bürger, C., Keller, F., \& Hautzinger, M. (2007). Reliability and validity of the revised Beck Depression Inventory (BDI-II). Results from the German samples. Nervenarzt, 78, 651-656.

Martiny, K., Refsgaard, E., Lund, V., Lunde, M., Thoughaard, B. Lindberg, L., \& Bech, P. (2015). Maintained superiority of chronotherapeutics vs. exercise in a 20-week randomized follow-up trial in major depression. Acta Psychiatrica Scandinavica, 131, 446-457.

Niederhofer, H., \& von Klitzing, K. (2011). Bright light treatment as add-on therapy for depression in 28 adolescents: A randomized trial. Primary Care Companion for CNS Disordorders, 13(6), 10.4088/PCC. $11 \mathrm{~m} 01194$.

Stephenson, K. M., Schroder, C. M., Bertschy, G., \& Bourgin, P., 2012. Complex interaction of circadian and non-circadian effects of light on mood: Shedding new light on an old story. Sleep Medicine Reviews, 16, 445-454.

Swedo, S.E., Allen, A.J., Glod, C.A., Clark, C.H., Teicher, M.H., Richter, D., ... Rosenthal, N. E. (1996). A controlled trial of light therapy for the treatment of pediatric seasonal affective disorder. Journal of the American Academy of Child and Adolescent Psychiatry, 36, 816-821.
Viola, A., Hubbard, J., Comtet, H., Hubbard, I., Delloye, E., Ruppert, E., \& Bourgin, P. (2014). Beneficial effect of morning light after one night of sleep deprivation: Light glasses versus light box administration (Poster). Society for Light Treatment and Biological Rhythms Annual Meeting, Vienna, Austria.

Wirz-Justice, A., Benedetti, F., \& Terman, M. (2013). Chronotherapeutics for affective disorders: A clinician's manual for light and wake therapy (2nd ed.). Basel: Karger.

Manuscript submitted: 09.02.2018

Accepted after revision: 25.05 .2018

Published online: 17.07.2018

Conflicts of interests:

Prof. Holtmann served in an advisory role for Shire and Medice, and received conference attendance support or was paid for public speaking by Lilly, Medice, Neuroconn, and Shire. He receives research support from the German Research Foundation and the German Ministry of Education and Research.

He receives royalties as Editor-in-Chief of the German Journal for Child and Adolescent Psychiatry and for textbooks from Hogrefe. Prof. Legenbauer received conference attendance support from Lilly as well as royalties for textbooks from Hogrefe and Springer. The present work is unrelated to the above grants and relationships.

The other authors have no conflicts of interest.

\section{Inken Kirschbaum-Lesch}

Ruhr University Bochum

LWL-University Hospital for Child and Adolescent Psychiatry Heithofer Allee 64

59071 Hamm

Germany

inken.kirschbaum@rub.de 